\title{
Embryo viability, duration of gestation and birth weight in sheep after transfer of in vitro matured and in vitro fertilized zygotes cultured in vitro or in vivo
}

\author{
P. Holm ${ }^{1,3}$, S. K. Walker ${ }^{2}$ and R. F. Seamark ${ }^{1}$ \\ ${ }^{1}$ Department of Obstetrics and Gynaecology, University of Adelaide, Adelaide, SA 5005, Australia; \\ ${ }^{2}$ South Australian Research and Development Institute, Turretfield Research Centre, Rosedale, SA 5350, \\ Australia; and ${ }^{3}$ Embryo Technology Center, Danish Institute of Animal Science, DK-8830 Tjele, Denmark
}

\begin{abstract}
The influence of various in vitro procedures on embryo survival and the production of normal offspring was investigated in sheep. Zygotes produced from in vitro matured (IVM) and fertilized (IVF) oocytes derived from slaughtered Merino ewes were allocated to three culture treatments for 6.5 days. Two groups were cultured in vitro in the absence or presence of oviduct epithelial cells while the third group was cultured in vivo in the oviducts of synchronized ewes. A fourth group of zygotes obtained from superovulated Merino ewes was also cultured in vivo and served as controls. After culture, IVM-IVF morulae and blastocysts, and control embryos were transferred to final recipient ewes. Pregnancy was diagnosed at day 50 of gestation by ultrasonography and pregnancies were allowed to go to term. The survival to term of IVM-IVF zygotes cultured in vitro was reduced compared with both in vivo cultured IVM-IVF zygotes and control zygotes (25-35\% versus $51-60 \%$, respectively, $P<0.05$ ). Day 6.5 IVM-IVF morulae had a lower survival rate than did control morulae regardless of culture treatment $(P<0.05)$, while survival rates of day 6.5 IVM-IVF blastocysts cultured in vivo did not differ from those of control blastocysts $(P>0.1)$. Both the gestation period and birth weight of IVM-IVF lambs were increased when compared with controls, the former significantly in all groups (154.0-154.9 days versus 150.6 days; $P<0.01$ ), while the latter increase was on the borderline of significance $(4.5-4.8 \mathrm{~kg}$ versus $4.0 \mathrm{~kg} ; 0.01 \leq P \leq 0.1$, respectively) and dependent on the prolongation of the gestation period. It is concluded that in vitro maturation and fertilization compromise subsequent embryonic and fetal development in sheep irrespective of the subsequent in vivo or in vitro culture treatment. Subjecting IVM-IVF zygotes to in vivo culture for 6.5 days minimizes only some of these effects, thus leading to the aberrant production of some offspring.
\end{abstract}

\section{Introduction}

Successful embryo culture plays an important role in the development of techniques involved in the multiplication of genetically superior or rare genotypes. These techniques can include in vitro maturation (IVM) and fertilization (IVF) of oocytes, use of co-culture of embryos with somatic cells (for example, oviduct epithelial cells) and collection of embryos from superovulated animals. However, the viability in vitro and in vivo of embryos produced or cultured in vitro is affected adversely (Czlonkowska et al., 1991; Kelk et al., 1992; Walker et al., 1992a; Holm et al., 1994a), and this is a major constraint to further developing the technologies. In addition, Walker et al. (1992a) observed that in vitro culture resulted in prolonged gestation, increased birth weight and increased perinatal death rate after embryo transfer. Similar findings have been reported in sheep (Thompson et al., 1995) and in cattle (Behboodi et al, 1995; Farin and Farin, 1995).

Revised manuscript received 18 March 1996.
Factors responsible for either the reduced viability of embryos or the production of abnormal offspring after in vitro handling have not been identified. The purpose of the present study was to examine the effect of different in vitro procedures on the rates of embryo survival and the production of normal offspring after embryo transfer. Specifically, the effects of the following factors were compared: (1) source of embryos (IVM-IVF versus in vivo derived), (2) in vitro versus in vivo culture of in vitro derived zygotes and (3) co-culture versus non-co-culture of in vitro derived zygotes.

Preliminary results have been published earlier in abstract form (Holm et al., 1994b).

\section{Materials and Methods}

Experimental design (Fig. 1)

In six replicates conducted from September to December 1992, zygotes produced by IVM-IVF were cultured for 
Treatments

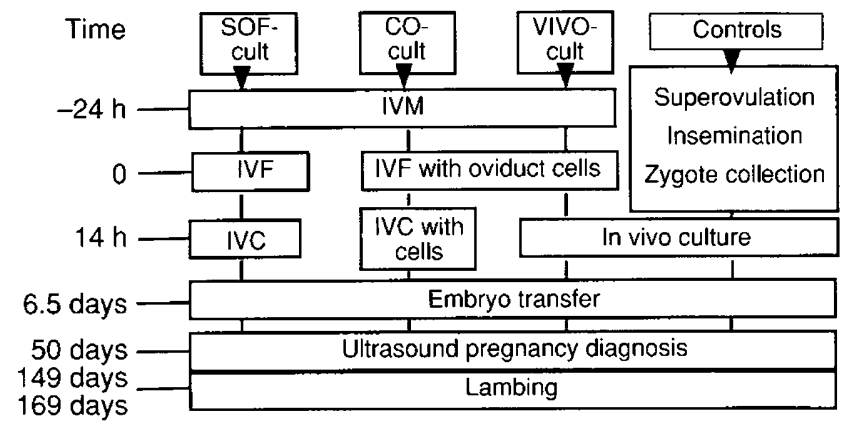

Fig. 1. Experimental design illustrating the time of in vitro maturation (IVM), in vifro fertilization (IVF) with or without ovine oviduct epithelial cells, and in vitro culture (IVC) in synthetic oviduct fluid with (SOF-cult) or without (CO-cult) oviduct cells or in vivo culture (VIVO-cult) in oviducts of synchronized sheep for the three IVM-IVF treatment groups and one control group in which zygotes were collected from superovulated ewes $16-20 \mathrm{~h}$ after the expected median time of ovulation.

6.5 days under three different conditions; SOF-cult: in vitro culture in cell-free synthetic oviduct fluid (SOF; Tervit et al., 1972 ) with $20 \%$ heat-inactivated human serum (HS), CO-cult: in vitro culture in SOF in the presence of ovine oviduct epithelial cells, and VIVO-cult: in vivo culture in non-ligated oviducts of synchronized ewes. In vivo derived zygotes (control) were subjected to culture in vivo for 5.5 days (approximately 6 days after the expected median time of ovulation). All embryos were transferred to synchronized recipients after the respective culture period. Recipient ewes were treated such that their expected median time of ovulation was within $12 \mathrm{~h}$ of the time of ovulation in donor ewes or the time of IVF.

\section{Production of in vivo derived zygotes}

South Australian Merino sheep located at Turretfield Research Centre were treated according to routine procedures for collection and transfer of embryos (Walker et al., 1986). Briefly, donor ewes were treated with progestagen pessaries (60 mg medroxyprogesterone acetate; Upjohn, Rydalmere) for 12 days and $17 \mathrm{mg}$ FSH (FSH-P: Heriot Agvet Pty Ltd, Melbourne, Victoria) administered as two daily injections i.m. approximately $12 \mathrm{~h}$ apart $(5,4,3,3$ and $2 \mathrm{mg})$, starting $48 \mathrm{~h}$ before pessary removal. At $27 \mathrm{~h}$ after pessary removal, $25 \mu \mathrm{g}$ synthetic GnRH (Intervet, Artamon, NSW) was injected i.m., followed $18 \mathrm{~h}$ later by laparoscopic insemination (Killeen and Caffery, 1982) of frozen-thawed semen pooled from three rams.

Zygotes were collected by mid-ventral laparotomy $12-15 \mathrm{~h}$ after the expected median time of ovulation. A Tom Cat catheter (Sherwood Medical, St Louis, MO) was inserted into the fimbriated end of the oviduct and $10 \mathrm{ml}$ of Dulbecco's PBS with $2 \%$ sheep serum (mPBS) was flushed through the oviduct from the tip of the uterine horn. Zygotes were washed and stored in SOF $+20 \%$ HS for up to $4 \mathrm{~h}$ before being transferred to intermediate recipients.
Production of in vitro derived zygotes

Zygotes were produced using the procedures of Holm $e t$ al. (1994a). Briefly, ovaries were collected from nonpregnant South Australian Merino ewes slaughtered at a local abattoir. Immature oocytes were aspirated from $2-4 \mathrm{~mm}$ follicles and those with evenly granulated ooplasm surrounded by tight cumulus cells were selected for IVM. During aspiration and selection, oocytes were kept at $30-35^{\circ} \mathrm{C}$ in Hepes-buffered TCM-199 (Gibco, Melbourne, Victoria) supplemented with 2\% heat-inactivated sheep serum (SS) and $25 \mathrm{iu}$ heparin $\mathrm{ml}^{-1}$ (H-3393, Sigma, MO). The oocyte-cumulus complexes were matured in bicarbonate-buffered TCM-199 supplemented with $15 \%$ heat-inactivated fetal calf serum (Commonwealth Serum Laboratories, Melbourne, Victoria), $5 \mu \mathrm{g} \quad \mathrm{FSH} \quad \mathrm{ml}^{-1}$ (Folltropin-V: Vetrepharm Inc., Melbourne, Victoria), $5 \mu \mathrm{g} \mathrm{LH}$ $\mathrm{ml}^{-1}$ (Lutropin-V: Vetrepharm) and $1 \mu \mathrm{g}$ oestradiol $\mathrm{ml}^{-1}$ (E-2257: Sigma) for $24 \mathrm{~h}$. Thereafter, cumulus cells were removed by gentle pipetting in $0.1 \%(\mathrm{w} / \mathrm{v})$ hyaluronidase (H-3506: Sigma) and ova were randomly allocated to three treatment groups for fertilization in SOF $+20 \%$ SS either without (experimental group SOF-cult) or with ovine oviduct epithelial cells (experimental groups CO-cult and VIVO-cult). Ova were incubated with frozen-thawed semen for $14 \mathrm{~h}$ at a final concentration of $1.5 \times 10^{\circ}$ motile spermatozoa ml $\mathrm{m}^{-1}$ in $50 \mu \mathrm{l}$ drops of medium under paraffin oil (10-15 ova per drop) in an atmosphere of $5 \% \mathrm{CO}_{2}: 5 \% \quad \mathrm{O}_{2}: 90 \% \mathrm{~N}_{2}$. The thawed semen pellets were subjected to a $1.5 \mathrm{~h}$ swim-up procedure and the motile fraction was added directly to the fertilization drops. The semen used for IVF was collected and pooled from the same rams that provided semen for laparoscopic insemination.

\section{Culture procedures}

After three washes in Hepes-buffered SOF $+20 \% \mathrm{HS}$, in vitro produced zygotes were cultured for 6 days either in SOF $+20 \%$ HS in the absence (SOF-cult) or presence of ovine oviduct epithelial cells (CO-cult) or in non-ligated oviducts of synchronized ewes (VIVO-cult). In vitro culture occurred in an atmosphere of $5 \% \mathrm{CO}_{2}: 5 \% \mathrm{O}_{2}: 90 \% \mathrm{~N}_{2}$ at $38.5^{\circ} \mathrm{C}$ in $50 \mu \mathrm{l}$ drops containing 12-15 embryos. In vitro culture medium and oviduct epithelial cells were changed every $48 \mathrm{~h}$. At the end of the culture periods, morulae and blastocysts from in vitro produced zygotes and all recovered in vivo produced embryos were washed and held in Hepes-buffered SOF $+20 \%$ HS for up to $3 \mathrm{~h}$ at $38.6^{\circ} \mathrm{C}$ until transfer.

\section{Preparation of ovine oviduct epithelial cell co-culture}

Oviduct epithelial cell co-cultures were prepared as described by Holm et al. (1994a). In summary, oviducts of post-oestrous sheep were freed from ligaments and adjoining blood vessels and $1.5 \mathrm{~cm}$ of both the ampulla and isthmus regions were removed. Epithelial cells were expressed into a Petri dish with Hepes-buffered TCM-199 and segregated into small clusters by flushing through a 30 gauge needle. The cell suspension was then washed by centrifugation (three times $500 \mathrm{~g}$ for $5 \mathrm{~min}$ ). After the final spin, the soft pellet was diluted 1:I and $10 \mu \mathrm{l}$ of this cell suspension were transferred to $1 \mathrm{ml}$ of bicarbonate buffered TCM-199 +20\% SS and cultured overnight at $38.6^{\circ} \mathrm{C}$ 
in $5 \% \mathrm{CO}_{2}$ in air. The cell clusters were then washed three times in either IVF- or IVC-medium and transferred to each of the IVF- or IVC-culture drops (approximately 150 cell clusters per $50 \mu \mathrm{l}$ drop). The oviduct epithelial cells were added $6 \mathrm{~h}$ before the start of co-culture and new co-culture drops were made every $48 \mathrm{~h}$ from the primary cell culture.

\section{Embryo transfer procedures}

Intermediate and final recipients were treated with progestagen pessaries ( $60 \mathrm{mg}$; Upjohn) for 12 days and 400 iu pregnant mares' serum gonadotrophin (Intervet) was administered at the time of pessary removal.

Presumptive in vivo and in vitro zygotes were transferred by mid-ventral laparotomy to intermediate recipients approximately $12 \mathrm{~h}$ after the expected median time of ovulation. Transfer was to a nonligated oviduct ipsilateral to an ovary containing at least one corpus luteum; embryos were in groups of 10-30 per ewe. Embryos were subsequently recovered by mid-ventral laparotomy at the end of the culture period. A Foley catheter (size 10 FG, Sherwood Medical) was inserted into the uterine lumen and the oviduct and uterine horn were flushed with $20 \mathrm{ml}$ mPBS. Recovered embryos were washed and stored in SOF $+20 \%$ HS for $2-4 \mathrm{~h}$ before being transferred to final recipients.

Embryos produced in vivo and in vitro were transferred laparoscopically on day $6-6.5$ to the tip of a uterine horn ipsilateral to an ovary with at least one corpus luteum. Blastocysts produced in vitro were transferred in pairs or, in two instances, in groups of three. In vitro produced morulae were transferred in groups of 5-13 per recipient as previous studies have demonstrated unequivocally that embryos that had not developed beyond the morula stage after 5 days in vitro culture were not viable or of very low viability ( $\mathrm{S}$. $\mathrm{K}$. Walker, unpublished). Consequently, by transferring these large numbers of embryos per ewe, worthwhile savings in the use of animals were made without the likelihood of producing litter-bearing pregnancies. All but one of the in vivo derived embryos were transferred in pairs. If too few recipients were available on a given day, in vitro derived embryos were selected for transfer so that the proportion transferred from each experimental group was similar to the proportion that had developed in that experimental group on that particular day.

All surgeries in this study were conducted under general anaesthesia induced by $10 \%(\mathrm{w} / \mathrm{v})$ thiopenthane sodium (Pentothal: Boehringer Ingelheim, Artamon) and maintained by a mixture of halothane (May and Baker, Dagenham) and oxygen.

Animal experimentation was performed in accordance with the Australian Code of Practice for the Care and Use of Animals for Scientific Purposes and the Prevention of Cruelty to Animals Act 1985 after approval was granted by the Animal Ethics Committees of both the South Australian Research and Development Institute and the University of Adelaide.

\section{Pregnancy and lambing observations}

Pregnancies and numbers of fetuses were determined at day 50 of gestation by ultrasonography with a Microimager 1000 sector ultrasound scanner equipped with a $3.5 \mathrm{MHz}$ abdominal transducer (Ausonics, Sydney). Pregnancies were allowed to go to term. Each ewe was identified with a side-brand number. Individual ewe-lamb details were recorded at lambing. The number, date of birth, birth weight and sex of lambs born were recorded along with incidences of assisted births and perinatal deaths. The flock was observed in both the morning and afternoon of each day to minimize the likelihood of mismothering occurring during the recording period.

\section{Statistical analyses}

Chi-squared tests were used to examine differences in developmental rates of in vitro produced ova.

Pregnancy rates and fetal survival rates at 50 days of gestation and at term were analysed by a mixed linear model (SAS Institute Inc., 1992). The model included culture treatment, replicate, embryo stage at transfer (when applicable), pregnancy type (twin/single) and sex of lamb as fixed effects and final recipient nested within replicate, experimental group, type of pregnancy and sex of lamb as a random effect.

Durations of gestation and birth weights were initially analysed in the same way using same variants as fixed effects, but variants with no effect $(P>0.2)$ were omitted in the final analysis. In addition, birth weight data were analysed both with and without duration of gestation as an additional fixed effect. If fixed effects were significant $(P<0.05)$, linear contrasts were used to determine the significance $(P<0.05)$ of the individual levels within the fixed effect. Results are expressed as least squares means (LS means) \pm SEM.

\section{Results}

\section{Embryo development and survival}

A total of 328 IVM-IVF embryos were transferred to 110 recipient ewes. Ninety-four ewes received 190 blastocysts and 16 ewes received 138 morulae. Sixty-three in vivo embryos ( 24 blastocysts, 29 morulae and ten earlier stages) derived from ten donors were transferred to 32 recipients.

Seven recipients were excluded from the data. One recipient died a week after transfer of 13 in vivo cultured IVM-IVF morulae and six pregnant recipients were missing at term. The latter ewes received two in vivo cultured IVM-IVF blastocysts (one ewe), two in vitro cultured IVM-IVF blastocysts (one ewe) and seven in vivo control zygotes (four ewes).

Culture treatment did not affect the proportions of morulae (14-18\%) and blastocysts (28-33\%) that developed from IVM-IVF ova ( $n=448,503$ and 284 for SOF-cult, CO-cult and VIVO-cult, respectively) but a lower proportion of blastocysts had hatched in vivo by day 6.5 compared with in vitro (15\% versus $19 \%$ versus $5 \%$ for SOF-cult, $\mathrm{CO}$-cult and VIVO-cult, respectively; $P<0.001$ ). In contrast, none of the in vivo control blastocysts $(n=24)$ had hatched and only $17 \%$ were expanded at the time of transfer.

The survival rates of morulae and blastocysts after transfer are shown (Fig. 2). The number of embryos surviving to day 50 of gestation and term were affected by culture treatment ( $P=0.0002$ and $P=0.0025$, respectively). The survival of 


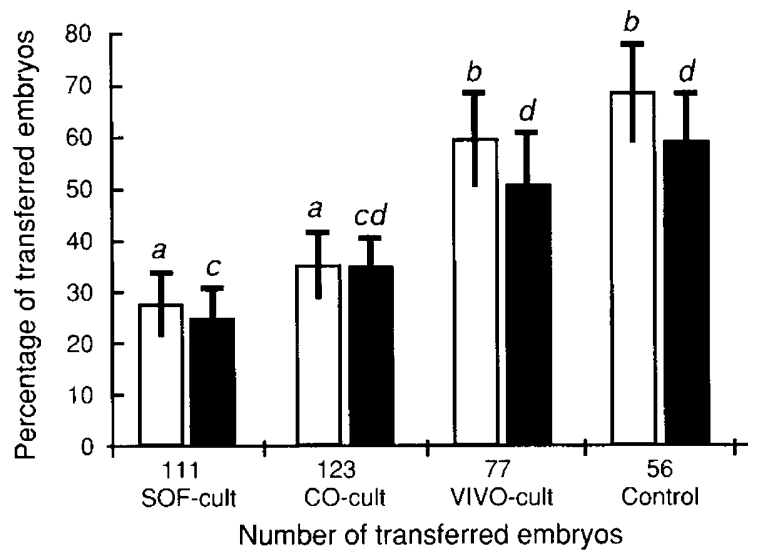

Fig. 2. Overall embryo survival rates (calculated as LS means \pm SEM) at 50 days of gestation $(\square)$ and at term ( $(\mathbf{C})$ after transfer of IVM-IVF morulae and blastocysts cultured for 6.5 days after IVF in SOF in the absence (SOF-cult) or presence of ovine oviduct (CO-cult), or in vivo (VIVO-cult), or transfer of in vivo control embryos (control) at day 6 after the estimated median time of ovulation. Data are statistically analysed by a mixed linear model using culture treatment, replicate, embryo stage as fixed effects and recipient as a random effect. $a b$ and $c d: P<0.05$ for different superscript within similar bars.

IVM-IVF zygotes cultured in vitro was significantly poorer than that of control zygotes, regardless of the presence or absence of oviduct epithelial cells. There was no significant difference between the overall survival rates of in vivo cultured IVM-IVF zygotes and in vivo produced zygotes.

As expected, the viability of IVM-IVF morulae was low with no signs of overcrowding of fetuses observed at ultrasound scanning at day 50 of gestation; two single lambs were obtained from 91 in vitro cultured morulae transferred in batches of 6-12 to each of 10 recipients, and three single lambs and one pair of twins were born from 28 in vivo cultured morulae transferred in batches of five to four recipients and eight to one recipient, respectively. However, the transfer of 20 in vivo derived morulae in pairs resulted in the birth of ten lambs. The transfer of earlier stage in vivo embryos as pairs $(n=2)$ or accompanying an in vivo morulae or blastocyst $(n=8)$ resulted in one single lamb and six lambs (e.g. four singles, one set of twins), respectively.

The developmental stage of embryos on the day of transfer influenced the survival rate of in vitro produced embryos $(P<0.001)$ but not of in vivo produced embryos (Fig. 3); the viability of day 6.5 IVM-IVF morulae was reduced compared with that of the IVM-IVF blastocysts $(P<0.008)$. Yet, analysis of the viability of specific stages of IVM-IVF blastocysts (Table 1) revealed that all blastocyst stages survived equally well to term when cultured in vivo or in the presence of oviduct cells while early (that is, delayed) blastocysts cultured in vitro in the absence of oviduct cells survived poorly compared with expanded and hatched blastocysts within the same treatment group. Furthermore, in all IVM-IVF treatment groups, hatched blastocysts survived equally well, while this was not the case for early and expanded blastocysts.

The fetal loss after day 50 of gestation varied substantially among treatment groups (SOF-cult: $9 \%(2$ of 23 ) versus CO-cult: $0 \%$ ( 0 of 39 ) versus VIVO-cult: $15 \%$ ( 6 of 41 ) versus control: $20 \%$ ( 7 of 35$)$ ). The fetal loss in the control group was
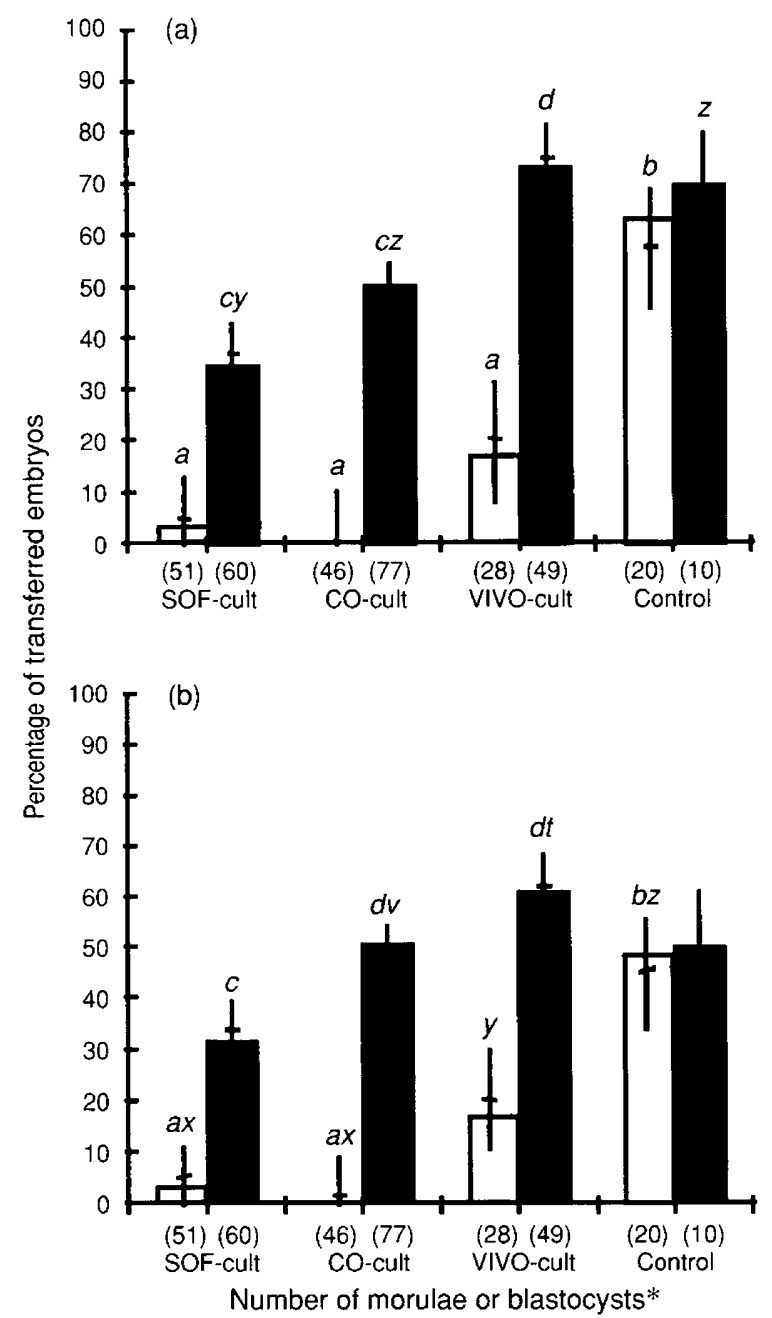

Fig. 3. Embryo survival (a) at 50 days of gestation and (b) at term, after transfer of IVM-IVF morulae ( $\square$ ) and blastocysts ( $\boldsymbol{\square}$ ) cultured for 6.5 days after IVF in SOF medium in the absence (SOF-cult) or presence of ovine oviduct cells (CO-cult), or in vivo (VIVO-cult), or transfer of in vivo control embryos (control) at day 6 after the estimated median time of ovulation. The pin-bars represent LS means - \% \pm SEM calculated by a mixed linear model using culture treatment, replicate, embryo stage as fixed effects and recipient as a random effect. $P<0.05$ for $a b$ and $c d$ and $P<0.1$ for $v t$ and $x y z$ within similar bars. ${ }^{*}$ Transferred in pairs or groups of identical embryonic stages.

high compared with the approximately $10 \%$ usually seen in comparable experimental groups at Turretfield Research Centre.

\section{Gestation period and birth weight of lambs}

A total of 95 IVM-IVF lambs and 28 control lambs were born and the sex ratio (females:males) for each group was 0.51 $(48: 46)$ and 0.46 (13:15), respectively. One lamb was predated before sex and weight was determined. Birth weights and duration of gestation of individual lambs are shown (Fig. 2).

The number of single and twin births in the four experimental groups were, respectively, 15 and three for the 
Table 1. Embryo survival rates (least squares means $\% \pm$ SEM) at term after transfer of ovine IVM-IVF derived morulae and blastocysts cultured in cell-free medium (SOF-cult), co-cultured with ovine oviduct cells (CO-cult) or cultured in vivo (VIVO-cult)

\begin{tabular}{lcccc}
\hline & \multicolumn{3}{c}{ Stage of blastocysts at transfer } & $\begin{array}{c}\text { Probability } \\
\text { for effect } \\
\text { of stage }\end{array}$ \\
\cline { 2 - 5 } Culture treatment & Early & Expanded & Hatched & $\begin{array}{c}\text { (1) } \\
\text { SOF-cult }\end{array}$ \\
CO-cult & $6.7 \pm 11.0(16)^{\mathrm{a}}$ & $39.4 \pm 8.5(26)^{\mathrm{c}}$ & $48.8 \pm 10.4(16)$ & $P<0.02$ \\
VIVO-cult & $38.6 \pm 15.2(19)^{\mathrm{ab}}$ & $40.5 \pm 7.8(36)^{\mathrm{c}}$ & $60.0 \pm 7.9(22)$ & $P>0.05$ \\
& $69.4 \pm 17.7(6)^{\mathrm{b}}$ & $67.5 \pm 7.4(36)^{\mathrm{d}}$ & $44.5 \pm 30.4(7)$ & $P>0.05$ \\
\hline
\end{tabular}

(n), number of blastocysts transferred in six replicates as singles or in pairs of same developmental stage. Different superscript within columns: $P<0.05$ for ab and $c d$.

Statistics: least squares mean-values are calculated with a mixed linear model using culture treatment, replicate, embryo stage as fixed effects and recipient within these as a random effect. Replicate effect was not significant.

Table 2. Duration of gestation and birth weight of lambs born after transfer of day 6.5 IVM-IVF embryos cultured in cell-free medium (SOF-cult), co-cultured with ovine oviduct cells (CO-cult) or cultured in vivo (VIVO-cult), and of in vivo control embryos (Control)

\begin{tabular}{|c|c|c|c|c|c|c|c|c|c|c|c|c|}
\hline \multirow{3}{*}{$\begin{array}{l}\text { Treatment } \\
\text { group }\end{array}$} & \multirow{2}{*}{\multicolumn{2}{|c|}{$\begin{array}{c}\begin{array}{c}\text { Duration of } \\
\text { gestation } \\
\text { (LS mean) }\end{array} \\
\text { Overall }\end{array}$}} & \multicolumn{10}{|c|}{ Birth weight (LS means) } \\
\hline & & & \multicolumn{2}{|r|}{ Overall } & \multicolumn{2}{|r|}{ Singles } & \multicolumn{2}{|r|}{ Twins } & \multicolumn{2}{|c|}{ Females } & \multicolumn{2}{|r|}{ Males } \\
\hline & $n$ & days & $n$ & $\mathrm{~kg}$ & $n$ & $\mathrm{~kg}$ & $n$ & $\mathrm{~kg}$ & $n$ & $\mathrm{~kg}$ & $n$ & $\mathrm{~kg}$ \\
\hline SOF-cult & 21 & $154.9 \pm 0.4^{* *}$ & 21 & $4.7 \pm 0.3^{*}$ & 15 & $5.2 \pm 0.3^{*}$ & 6 & $4.4 \pm 0.5$ & 11 & $4.5 \pm 0.2$ & 10 & $5.1 \pm 0.3$ \\
\hline CO-cult & 39 & $154.0 \pm 0.5^{* *}$ & 39 & $4.5 \pm 0.2^{\dagger}$ & 17 & $5.5 \pm 0.3^{* *}$ & 22 & $3.5 \pm 0.3$ & 18 & $4.4 \pm 0.2$ & 21 & $4.6 \pm 0.3$ \\
\hline VIVO-cult & 35 & $154.2 \pm 0.5 * *$ & $34^{\mathrm{a}}$ & $4.8 \pm 0.2^{* *}$ & 18 & $5.2 \pm 0.3^{*}$ & 16 & $4.4 \pm 0.3$ & 19 & $4.5 \pm 0.3$ & 15 & $5.1 \pm 0.3$ \\
\hline Control & 28 & $150.6 \pm 0.5$ & 28 & $4.0 \pm 0.2$ & 22 & $4.5 \pm 0.2$ & 6 & $3.9 \pm 0.5$ & 13 & $3.8 \pm 0.2$ & 15 & $4.5 \pm 0.4$ \\
\hline Probability for treatment effect & & $P<0.01$ & & $P=0.05$ & & $P=0.05$ & & $P=0.12$ & & $P=0.10$ & & $P>0.20$ \\
\hline
\end{tabular}

n, number of lambs.

*Different from control group by $P \leq 0.05 ;{ }^{* *}$ Different from control group by $P<0.01$; ${ }^{\dagger}$ Different from control group by $P=0.1$. The significant effect of treatment on birth weight is not seen if duration of gestation is included as co-variate in the statistical model.

ane newborn lamb predated weighing.

Statistics: LS mean values are calculated with a mixed linear model using culture treatment, sex of lamb and single/twin birth as main effects and randomizing recipient effects within these. No interactions were found between tested parameters.

SOF-cult-group, 17 and 11 for the CO-cult-group, 18 and eight for the VIVO-cult-group and 22 and three for the control group.

The rates of assisted births and perinatal death of lambs were 0 of 21 and 0 of 21 for the SOF-cult-group, 6 of 39 and 8 of 39 for the CO-cult-group, 1 of 34 and 2 of 34 for the VIVO-cult-group and 1 of 28 and 0 of 28 for the control group, respectively.

The mean gestation period in the SOF-cult, CO-cult and VIVO-cult treatment groups did not differ significantly (154.0154.9 days). However, the mean gestation period in the control group was 4 days shorter ( 150.6 days; $P<0.01$ ) (Table 2). The mean duration of gestation did not vary significantly between dams with either female or male lambs (153.0 days for both sexes). Single and twin lambs were born after $153.5 \pm 0.3$ days and $152.5 \pm 0.4$ days, respectively $(P=0.09)$. Neither day of transfer, nor developmental stage of the embryo significantly influenced gestation length $(P>0.50)$.
The birth weight of lambs was influenced by single/twin pregnancy, sex and culture treatment. The overall mean weights $(\mathrm{kg})$ of single versus twin lambs and female versus male lambs were $5.1 \pm 0.2$ versus $3.9 \pm 0.2(P<0.01)$ and $4.3 \pm 0.2$ versus $4.7 \pm 0.1(P=0.04)$, respectively.

All IVM-IVF lambs other than those derived from co-cultured embryos were significantly heavier than in vivo control lambs $(P \leq 0.05$ and $P=0.1$, respectively); the mean birth weights in the SOF-cult, CO-cult, VIVO-cult and control treatment groups were $4.7 \pm 0.4,4.5 \pm 0.2,4.8 \pm 0.2$ and $4.0 \pm 0.2$, respectively. Separate analysis of birth weights of singles and twins or females and males revealed significant differences between all treatment groups and controls for single lambs and tendencies for female lambs, respectively, while results were less clear for twin and male lambs, respectively (Table 2 and Fig. 4). There was no significant effect of culture treatment on birth weights when duration of gestation was included as fixed effect in the statistical model. 


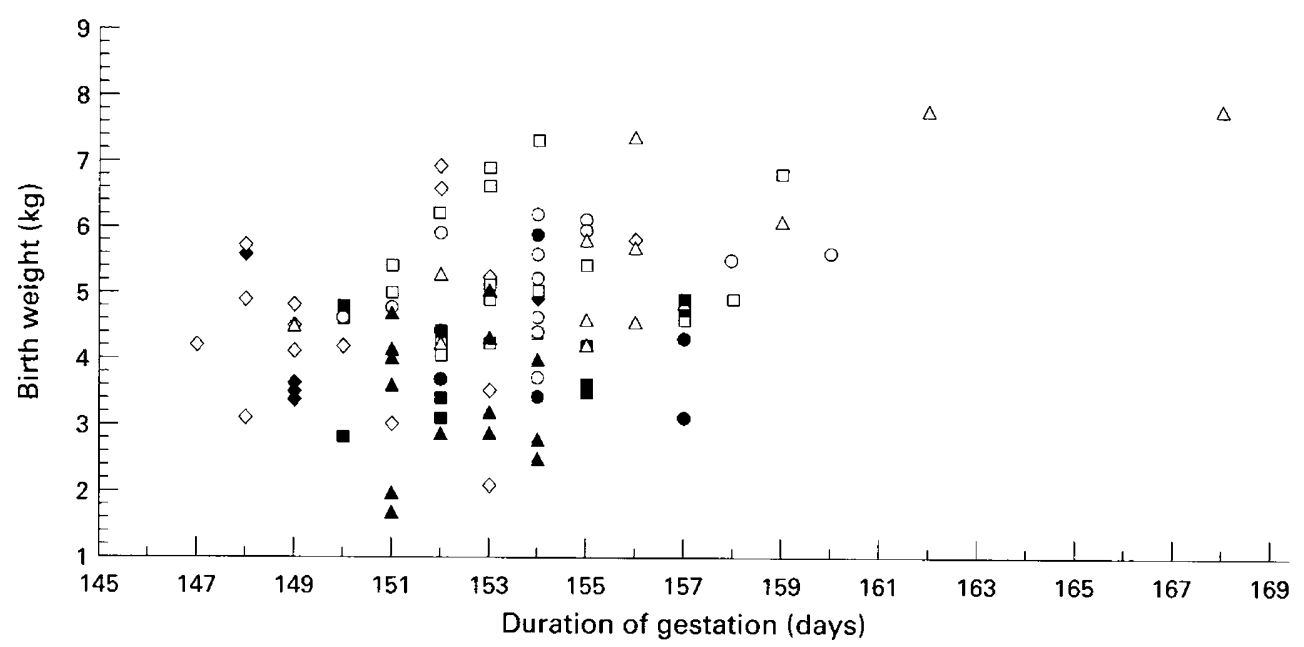

Fig. 4. Duration of gestation and birth weights of new-born single and $\mathbf{t w i n}$ lambs developed from IVM-IVF zygotes cultured for 6.5 days in synthetic oviduct fluid in the absence (singles: $\bigcirc$; twins: $)$ ) or presence of ovine oviduct cells (singles: $\triangle$; twins: $\boldsymbol{\Delta}$ ) or in vivo in nonligated oviducts of synchronized sheep (singles: $\square$; twins: $\mathbf{\square}$ ), or from control zygotes cultured in vivo as above (singles: $\diamond$; twins: $\bullet$ ).

\section{Discussion}

The in vitro procedures used in this study influenced not only the overall survival of embryos after transfer but also the duration of gestation and birth weight, thus supporting similar observations (Walker et al., 1992a, b; Thompson et al., 1995).

As in earlier studies (Holm et al., 1994a), the proportions of IVM-IVF zygotes that developed to the morula and blastocyst stages were not influenced by the absence or presence of oviduct cells or by the in vivo culture conditions. However, the in vitro environment influenced development, as more IVM-IVF blastocysts hatched during the culture period compared with in vivo culture and none of the in vivo derived control embryos hatched during their culture period. Similar findings have been reported by Walker et al. (1992a). This finding may be a result of the high serum contents in the culture medium, as recent publications have shown that the use of serum as the protein source in the culture medium may accelerate the early embryonic development when compared with albumin (Thompson et al., 1995; van Langendonckt et al., 1996).

Overall, in vitro culture of IVM-IVF zygotes for 6.5 days reduced embryo viability by $15-25 \%$ compared with in vivo culture; $25-35 \%$ of IVM-IVF-IVC morulae/blastocysts survived to term. These survival rates are higher than most comparable reports (Czlonkowska et al., 1991; Kelk et al., 1992), but lower than those reported by Thompson et al. (1994) who mainly transferred day 6 blastocysts. The oviduct and uterine environment of the synchronized intermediate recipient clearly improved the overall viability of IVM-IVF blastocysts, which was not different from that of in vivo produced control blastocysts. Our results indicate that slowly developing blastocysts (those that had just become blastocysts at day 6.5 after IVF) benefited most from an oviduct environment. Even the addition of oviduct cells to SOF tended to improve the viability of late forming blastocysts after transfer. This corresponds to the observations in sheep that the number of cells in blastocysts cultured in vitro are particularly low in the slowly developing blastocysts (Walker et al., 1992a; Holm et al., 1994a). There was also a tendency for a better survival of IVM-IVF morulae when they were cultured in vivo compared with in vitro, although they did not survive as well as their in vivo control morulae and no effects of adding oviduct cells to the SOF-medium were found. Previous studies of prolonged culture of in vivo produced zygotes (S. K. Walker, unpublished) suggested that IVM-IVF morulae produced after 6.5 days of culture also would be nonviable or have very low viability, and indeed we did not see any signs of fetal overcrowding at ultrasound scanning on day 50. Thus the results supported this presumption; only $30 \%$ of recipients became pregnant, and less than $5 \%$ of the IVM-IVF morulae survived to day 50 of gestation and to term.

The production of zygotes in vitro prolonged the average duration of gestation by 4 days compared with in vivo controls, irrespective of whether IVM-IVF embryos were cultured in vitro or in vivo. The overall mean birth weights of lambs derived from IVM-IVF embryos cultured either in vitro without oviductal cells or in vivo were also increased significantly compared with controls. Lambs derived from co-cultured IVM-IVF embryos tended to be heavier than controls, but only the mean birth weights of singles in this group were significantly heavier than controls. The increase in mean birth weights was not statistically independent of the duration of gestation, nor could the unusual heavy lambs derived from IVM-IVF zygotes be characterized as statistical outliers, as their gestation period also tended to be unusually long (Fig. 4). Therefore, the present results do not conclusively support the observations of Walker et al. (1992b) and Thompson et al. (1995) regarding independent increases in the duration of gestation and in birth weights after the in vitro culture of ovine zygotes in SOF with $20 \%$ serum.

However, the present data show that IVM-IVF procedures alone alter the duration of gestation, and as the birth is triggered by the fetus (Silver, 1990), the prolongation of gestation alone is likely to be a result of physiological changes in the fetus. 
The use of an oviduct epithelial cell co-culture system did not evidently normalize gestation length and birth weight. This finding is supported by studies in cattle (Behboodi et al., 1995; Farin and Farin, 1995). Also, the in vivo culture of in vitro produced zygotes failed to reverse these effects, although blastocyst survival rate was normalized. A parallel may be drawn to more radical manipulations to the oocyte (for example, nucleus transfer) that also cause disturbances in fetal growth and duration of gestation which are not normalized by in vivo culture (Willadsen et al., 1991; Wilson et al., 1995). Furthermore, unusually large offspring derived from IVM-IVF zygotes cultured in sheep oviducts have been reported in cattle, although the observed mean birth weight of the eight calves derived from this culture group did not differ from the 64 control artificial insemination calves (Behboodi et al., 1995).

Thompson et al. (1995) showed that duration of gestation and birth weight of lambs derived from both in vitro and in vivo produced zygotes were affected when the embryos were cultured in SOF with $20 \%$ human serum, but not in SOF in which sera were substituted with BSA and amino acids. This finding suggests that high contents of human serum in culture medium is a possible factor causing aberrant fetal development. All zygotes in the present study were subjected to high contents of human serum for at least a short period after fertilization. However, several other in vitro conditions may influence cytoplasmic and genetic factors in the oocyte and embryo important in fetal development (for review see Walker et al., 1996), and also the way in which the oocyte or embryo is manipulated may influence the final outcome. Exposure of early preimplantation embryos to aberrant conditions in vivo such as an asynchronous uterus (Wilmut and Sales, 1981) or to the oviducts of progesterone treated ewes (Kleemann et al., 1994) causes enhanced fetal growth. In cattle, in vitro procedures also affect fetal growth (Behboodi et al., 1995; Farin and Farin, 1995), but not duration of gestation (Behboodi et al., 1995), while cloning by nucleus transfer affects both (Willadsen et al., 1991; Wilson et al., 1995). The numbers of lambs and variation in birth weights in this study do not allow further conclusions regarding the possible relations between the type of manipulation, birth weight and duration of gestation.

The observation of physiological changes (for example abnormal metabolic regulation) in newborn nuclear transfer calves irrespective of their birthweight have been correlated with reduced neonatal survival (Garry et al, 1996). In vitro culture has also been linked with increased rates of perinatal death in both sheep (Walker et al., 1992a, b) and cattle (Behboodi et al., 1995). In the present study, we observed high incidence of perinatal death only in the group of lambs derived from co-cultured IVM-IVF zygotes. This group also had a high incidence of assisted births, an event that at least in part may explain the reduction in perinatal survival.

In conclusion, the results of our study indicate that duration of gestation and birth weight of lambs are increased when oocytes are subjected to IVM and IVF procedures. These effects are manifested before first cell division and influence development, regardless of the subsequent culture treatments. Neither the oviduct environment nor oviduct cells in culture were able to reverse these effects even though the survival rate of IVM-IVF produced blastocysts was normalized.
The authors thank B. A. Petersen and R. J. Ashman, University of Adelaide, for their surgical assistance and $\mathrm{H}$. Callesen, Embryo Technology Center, for his critical review of this manuscript. This work was supported by a grant from the Meat Research Corporation of Australia.

\section{References}

Behboodi E, Anderson GB, Bondurant RH and Cargill SL (1995) Birth of large calves that developed from in vitro-derived bovine embryos Theriogenology $44227-232$

Czlonkowska M, Eysymont U, Guszkiewicz A, Kossakowski M and Dziak J (1991) Birth of lambs after in vitro maturation, fertilization, and coculture with oviductal cells Molecular Reproduction and Development 30 34-38

Farin PW and Farin CE (1995) Transfer of bovine embryos produced in vivo or in vitro: survival and fetal development Biology of Reproduction 52 676-682

Garry FB, Adams R, McCann JP and Odde KG (1996) Postnatal characteristics of calves by nuclear transfer cloning Theriogenology 45 14I-152

Holm P, Irvine BJ, Armstrong DT and Seamark RF (1994a) Effect of epithelial cells on the fertilization and development of sheep oocytes in vitro Animal Reproduction Science 36 227-241

Holm P, Walker SK, Petersen BA, Ashman RJ and Seamark RF (1994b) In vitro versus in vivo culture of ovine IVM-IVF ova: effect on lambing Theriogenology 41217

Kelk DA, Gartley CJ and King WA (1992) Birth of lambs from embryos produced by in vitro maturation, fertilization and culture of oocytes Theriogenology 37237

Killeen ID and Caffery GJ (1982) Uterine insemination of ewes with the aid of a laparoscope Australian Veterinary Journal $\mathbf{5 9} 95$

Kleemann DO, Walker SK and Seamark RF (1994) Enhanced fetal growth in sheep administered progesterone during the first three days of pregnancy Journal of Reproduction and Fertility 102 411-417

SAS Institute Inc. (1992) The MIXED Procedure. In SAS Technical Report P-229. SAS/STAT Software: Changes and Enhancements. Release 6.07 pp 287-365. SAS Institute inc., Cary, NC

Silver M (1990) Prenatal maturation, the timing of birth and how it may be regulated in domestic animals Experimental Physiology 75 285-307

Tervit HR, Whittingham DG and Rowson LEA (1972) Successful culture in vitro of sheep and cattle ova Journal of Reproduction and Fertility 30 493-497

Thompson JGE, Gardner DK, Pugh PA, McMillam WH and Tervit HR (1995) Lamb birth weight following transfer is affected by the culture system used for pre-elongation development of embryos Biology of Reproduction 53 $1385-1391$

Van Langendonckt A, Auquier P, Donnay I, Massip A and Dessy F (1996) Acceleration of in vitro bovine embryo development in the presence of fetal calf serum Theriogenology $\mathbf{4 5} 194$

Walker SK, Smith DH and Seamark RF (1986) Timing of multiple ovulations in the ewe after treatment with FSH or PMSG with or without GnRH Journal of Reproduction and Fertility 77 135-142

Walker SK, Heard TM and Seamark RF (1992a) In vitro culture of sheep embryos without co-culture: successes and perspectives Theriogenology 37 111-126

Walker SK, Heard TM, Bee CA, Frensham AB, Warnes DM and Seamark RF (1992b) Culture of embryos of farm animals. In Embryonic Development and Manipulation in Animal Production pp 77-92 Eds A Lauria and F Gandolfi. Portland Press Ltd, London

Walker SK, Hartwich KM and Seamark RF (1996) The production of unusually large offspring following embryo manipulation: concepts and challenges Theriogenology 45 111-120

Willadsen SM, Janzen RE, McAlister RJ, Shea BF, Hamilton G and McDermand D (1991) The viability of late morulae and blastocysts produced by nuclear transplantation in cattle Theriogenology 35 161-170

Wilmut I and Sales DI (1981) Effect of an asynchronous environment on embryonic development in sheep Journal of Reproduction and Fertility 61 179-184

Wilson JM, Williams JD, Bondioli KR, Loney CR, Westhusin ME and McCalla DF (1995) Comparison of birth weight and growth characteristics of bovine calves produced by nuclear transfer (cloning), embryo transfer and natural mating Animal Reproduction Science 38 73-83 